\title{
The Use of Fitness Trackers to Increase the Endurance Indicator for People Over 50
}

\author{
Nagovitsyn R.S. \\ Department of Physical Culture and Life Safety \\ Glazov State Pedagogical Institute, \\ Glazov, Russia \\ gto18@mail.ru
}

\author{
Panachev V.D. \\ Department of Physical Culture \\ Perm National Research Polytechnic University, \\ Perm, Russia \\ panachev@pstu.ru
}

\author{
Legotkin A.N. \\ Department of Physical Culture \\ Perm National Research Polytechnic University \\ Perm, Russia \\ legotkin.pstu@gmail.com
}

\begin{abstract}
Background: the relevance of the training process for students of various ages using mobile devices has determined the research concept. Aim of the study: to identify and justify the use of fitness trackers to increase the stamina of people older than 50 years in running, mixed mobility and nordic walking. The participants of the present study middle-aged, elderly and senile people over 50 years old $(n=48)$. The respondents of the experimental group in an independent training process for the development of endurance used a mobile device - a fitness tracker connected to a smartphone. Using the gadget, the average athlete's heart rate was analyzed during aerobic exercise at a distance, and the optimal movement mode was set on the tracker. It controls the athlete's pace while overcoming the distance. Mathematical-statistical data processing was performed using Chi-square (X2) at $\mathbf{p}<0.05$. A statistical study of the results obtained over 6 months proved the effectiveness of using mobile devices when running and Nordic walking compared to overcoming the distance in the training process without using them. The revealed results reliably differ $(p<0.05)$ between focus groups in increasing the effectiveness of the distance running by athletes in terms of fulfilling the standards of the Ready for Labor and Defense sports complex. The use of mobile technology is more appropriate to use under the guidance of a coach and mainly for middle-aged athletes. Implementation of middle-aged, elderly and senile people cardiovascular system monitoring using mobile devices eliminates cases of overwork and injuries involved in active physical exercises.
\end{abstract}

Keywords-fitness tracker; endurance; running; nordic walking; middle-aged and elderly people

\section{INTRODUCTION}

Topical the problem of modern androgogy is the education of middle-aged, elderly and senile people on the use of mobile devices and innovative educational Internet-technologies in various areas of life. The modern mobile age, which is based on constantly updated technological capabilities, requires the presence of such qualities as mobility, the ability to work and be active. A harmonious combination of recreational, athletic and sports activities in physical, spiritual, moral and creative development, undoubtedly, contributes to the formation of such personality traits.

A preliminary study of the innovative technologies impact problem on the quality of fitness and fitness training for this age group showed that in this situation, contradictions are found:

- between a wide range of innovative surveys that guide the improvement of physical education and health training for middle-aged, elderly and senile people and the insufficient development of various training process methods based on the use of innovative information and communication technologies;

- between public awareness of the need for physical, intellectual and spiritual improvement of the individual, maintaining an optimal level of health through the formation of a person's physical culture and the lack of the necessary modern socio-pedagogical conditions for its formation;

- between a rapidly changing life, an increase in the pace of scientific and technological progress, the activation of social changes affecting people of different ages, who need to adapt to constantly changing living conditions, and the training process, which cannot fully respond to social changes and satisfy educational needs middle-aged, elderly and senile people.

The insufficient development of the methodological foundations for designing the training process on the basis of mobile devices allowed us to determine the purpose of the study - to identify and justify the use of fitness trackers to increase the stamina of people over 50 years of age in running, mixed movement and nordic walking. 
There are some recent studies explicitly proposing different types of the introduction of innovative technologies in the training process of middle-aged, elderly and senile people. In particular, research works aimed at individualizing sports training based on mobile technologies [1]. They consist in taking into account the individual structural qualification characteristics of those involved, in analyzing patterns and predicting the training process of athletes of different ages and their functional and physical characteristics and their physiological characteristics [2, 3]. Of particular note, studies on the implementation of information and mobile technologies in the physical education and sports process of athletes of various ages [4], including middle-aged, elderly and senile people [5]. And also, the use of various specially-developed mobile content for physical education and sports [6, 7]. But special attention was given to studies devoted to proving an increase in the effectiveness of the training process based on the introduction of information technologies of people over 50 $[8,9]$. An analysis of the scientific and methodological literature revealed particular relevance and novelty in the introduction of mobile devices, and allowed us to outline the vector of experimental work on testing the implementation of the fitness tracker $[10,11]$ in the process of engaging in anaerobic types of physical activity: running, mixed movement and nordic walking.

\section{MATERIALS AND Methods}

Participants: middle-aged, elderly and senile people over 50 years old $(n=48)$. Respondents corresponded to three age categories on the steps of the Russian sports complex «Ready for Labor and Defense», regardless of gender: IX stage - 50-59 years $(n=30)$, X stage - 60-69 years $(n=12)$, XI stage - over 70 years $(n=6)$. In turn, each group of participants was divided into two equal focus groups: experimental and control. During the experiment, all subjects underwent independent sports training according to the training program with the instructor's methodological recommendations to prepare for the implementation of the "Ready for Labor and Defense" standards: running (2000 meters), mixed movement (2000 meters) and nordic walking (3000 meters). However, the experimental group respondents used a mobile device, a fitness tracker connected to a smartphone, in an independent training process for running, mixed movement and nordic walking.

Materials: the theoretical and methodological materials used in the current study were implemented through the introduction of a mobile approach, the implementation of which, in conjunction with the system, individuallydifferentiated and active approaches, provides a higher quality result in the implementation of the training process in sports, in particular in aerobic types of physical activity. The solution of research problems was provided by a set of complementary theoretical methods for the analysis of domestic and foreign pedagogical theory, practice and experience in the field of fitness activities of the population of different ages; general scientific methods such as classification, modeling, comparison, comparison and generalization. Mathematicalstatistical data processing was performed using Chi-square (X2) at $p<0.05$. Monitoring the level of development of endurance was carried out using testing on the steps of the AllRussian physical culture and sports complex "Ready for Labor and Defense": IX stage - ha 2000 meters; IX-XI steps - Nordic walking for 3000 meters; IX-XI steps - mixed movement for 2000 meters. Testing performance was ranked by the following levels of performance from low to high: bronze, silver, gold.

Organization of the research: was focused on targeted stimulation of people of middle, elderly and senile age to independent physical culture and health-improving activities through individualization and differentiation of the use of mobile escort. The research process was achieved with the free provision of mobile technologies for each athlete of the experimental group, intensive physical education through mobile devices (smartphones). When using mobile content in the training process, it was especially important to identify the paramount, culture-forming, system-forming attention of students on the basic physical culture principles.

Before the experiment, the participants of both groups had a level of development of physical quality (endurance) that corresponded to an average below the standard of the bronze badge $(\mathrm{Xb})$ "Ready for Labor and Defense" equal to $\mathrm{Xb}^{*}(1.18 \pm 0.08)$ for all tests in tests running, mixed mobility and nordic walking. In turn, this proves that before the start of the experimental study, the participants in the study of the experimental and control groups were significantly identical $(\mathrm{p}>0.05)$.

For 6 months, respondents implemented an independent training process under the guidance of a mentor at least 3 times a week. Each sporting session for 50-60 minutes consisted of a general and aerobic warm-up, overcoming the track at the pace recommended by the trainer, and a dosage appropriate to the age / step "Ready for Labor and Defense": Monday / Tuesday - mixed movement of 2000 meters; Wednesday / Thursday - nordic walking 3000 meters; Friday / Saturday - running at a distance of $2000 \mathrm{~m}$ (for study participants over 70 years of age - mixed movement of 2000 meters), as well as special gymnastic exercises in the final part of the lesson. After the end of the first half of the experiment, the study participants were offered (not required) another lesson on Saturday / Sunday in terms of intensity and content of the training on Monday / Tuesday. The training process in the experimental group was accompanied by monitoring of the performance of a fitness tracker connected to a smartphone, and located on the athlete's arm during exercise. Using the gadget, the average heart rate at a distance was analyzed, and the optimal running mode was set on the tracker, which controls the athlete during the run using a special signal. Table of contents of training sessions for training weeks with the proposed mentor's optimal heart load and running speed, mixed movement and nordic walking in Table 1: 
gold badge (IX level "Ready for Labor and Defense") is significantly different at a confidence level of $\mathrm{p}<0.05$, which indicates the effect of the experiment on efficiency training process:

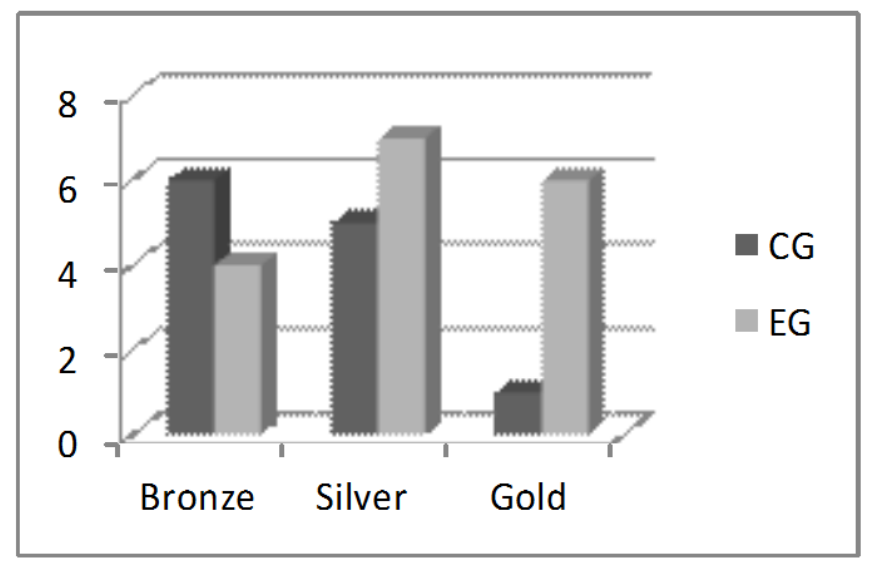

Fig. 1. Results of experimental work on testing the respondents' running

The results obtained indicate a positive result of experimental work on the effectiveness of the training process. It should be noted that a significant increase in the number of participants in the experimental group $(n=6)$ compared with the control group $(n=1)$ overcame the distance of 2000 meters in the gold insignia. Several $(n=3)$ participants in the study of the experimental group of a higher level took part in the control testing and showed a decent result for the bronze sign.

As shown in Figure 2, the number of participants in the EG and $\mathrm{CG}$ who completed the result in Nordic walking at a distance of 3000 meters (IX, X and XI steps "Ready for work and defense") for the bronze, silver and gold badges is significantly different at a confidence level at $\mathrm{p}<0.05$, which indicates the effect of the experiment on the effectiveness of the training process.

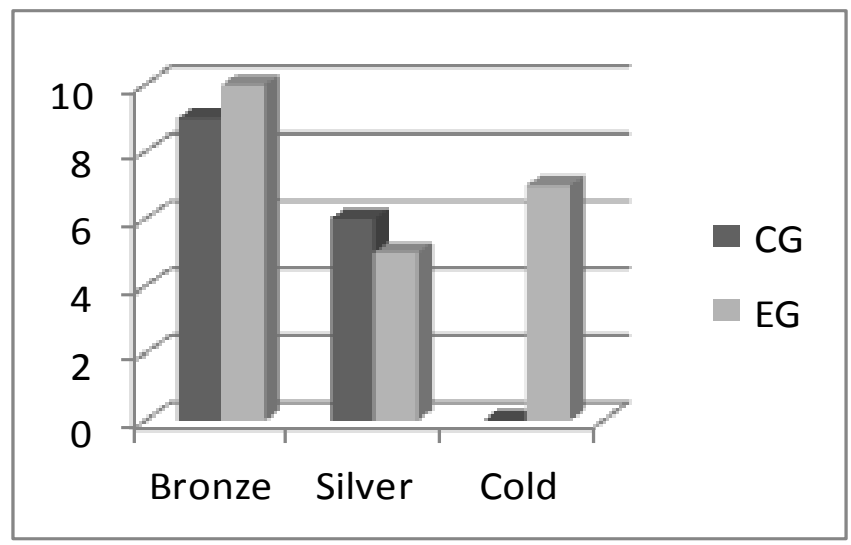

Fig. 2. Results of experimental work on testing nordic walking respondents

The recorded results reliably determine the effect of the experiment on the effectiveness of the training process on endurance. It should be noted that there is a significant increase in the number of participants in the experimental group $(n=7)$ compared to the control group in which no one 
completed the distance of 3,000 meters in the gold mark of distinction "Ready for Labor and Defense".

As shown in Figure 3, the number of EG and CG participants who performed the result in mixed movement to a distance of 3000 meters (X and XI steps "Ready for Labor and Defense") to the bronze, silver and gold badges was significantly the same at a confidence level at $\mathrm{p}>0.05$, which indicates an insufficient effect of the experiment on the effectiveness of the training process:

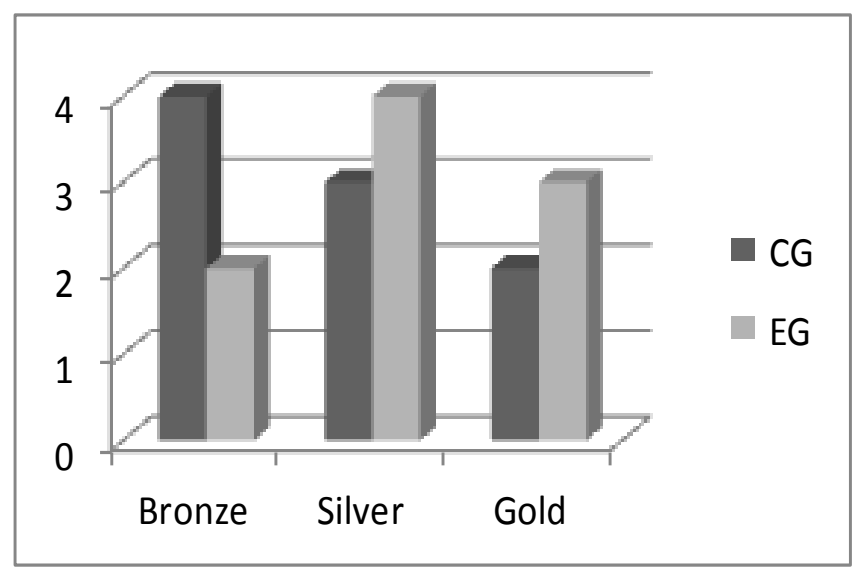

Fig. 3. Results of experimental work on testing mixed walking of respondents

The results obtained determine the effect of the experiment on the effectiveness of the training process on endurance. However, mathematical and statistical processing of the results of the study in mixed movement revealed not significant differences $(p>0.05)$ between the experimental and control groups. This result can be recorded due to the fact that the research group was quantitatively small $(n=18)$ and further research is needed. Also, the unreliable result obtained is due to the fact that both focus groups significantly increased the result in mixed movement. Mixed movement, which includes ordinary walking, is one of the most natural, familiar ways of moving a person. And the effectiveness in this automated motor act, carried out as a result of the complex coordinated activity of the skeletal muscles of the trunk and limbs, does not depend on the use of mobile devices. Systematic walking during any period of time, at any pace, any time of the day or any day of the week has a positive result.

\section{DISCUSSIONS}

In general, the results of this study are consistent with the results of other studies of the physical activity of athletes of different ages using innovative technologies [8, 12, 13]. A theoretical analysis of studies on the informatization of physical culture and sports activity proves that the regular use of these technologies increases the efficiency of the training process in various sports and physical activity of people of different ages [7, 14, 15]. Recently, many authors have proved that classes using mobile technologies are one of the effective conditions for the formation of a motivational-value attitude to physical education lifestyle $[16,17]$. Which ultimately leads to an increase in mass in sports and an increase in the level of physical development of the population [3, 18]. In research papers, the effectiveness of the use of mobile devices to develop the physical qualities of athletes of various qualifications is proved $[19,20]$. In turn, the author's study supplements the previous scientific developments with the results obtained. Namely, the use of mobile technologies, in particular fitness trackers [21], under the guidance of a trainer [11] and in a strictly regulated system of the training process, has a positive effect on running performance in those engaged in physical exercises $[10,22,23]$. However, the study proved that the older the athlete, the less effective the use of mobile devices in the development of endurance [24], compared with the training process without using these innovative technologies. Based on the obtained mathematical and statistical processing, it was proved in the work that the implementation of the training process using a fitness tracker connected to a smartphone significantly significantly improves the effectiveness of study participants over 50 years old, namely middle-aged, elderly and senile people.

\section{CONCLUSIONS}

Received statistics allow us to conclude that the use of mobile technologies is more appropriate to use under the guidance of a coach and mainly for middle-aged athletes. It should also be noted that the monitoring of the cardiovascular system of middle-aged, elderly and senile people using mobile devices allowed us to exclude cases of overwork and injuries of the study participants in the experimental group. Further research on the use of mobile devices in the training process will be aimed at studying their effect on the effectiveness of middle-aged athletes in terms of gender comparison. As well as other types of physical exercises and physical activity, such as cross-country skiing at 5000 meters and cross-country cross-country race at 3000 meters.

\section{References}

[1] I.E. Ioana, C.M. Mihaela, T. Mariana, G. Luminita, C. Constantin, The occupational diary as a smartphone application for monitoring the activities of daily living and physical exertion. Journal of Physical Education and Sport. vol. 18, 2018, pp. 2008-2013. doi: 10.7752/jpes.2018.s5298

[2] R.S. Nagovitsyn, P.B. Volkov and A.A. Miroshnichenko, Planning of physical load of annual cycle of students', practicing cyclic kinds of sports, training. Physical Education of Students, 2017, vol. 21 (3), pp. 126-33.

[3] C.C. Scarabottolo, E.S. Cyrino, P.M. Nakamura, W.R. Tebar, D.D.S Canhin, L.A. Gobbo and D.G. Christofaro, Relationship of different domains of physical activity practice with health-related quality of life among community-dwelling older people: A cross-sectional study BMJ Open, vol. 9, iss. 6, 2019, e02775. doi: 10.1136/bmjopen-2018-027751

[4] Z. Kozina, O. Ol'khovyj and V. Temchenko, Influence of information technologies on technical fitness of students in sport-oriented physical education. Physical Education of Students, vol. 20 (1), 2016, pp. 21-28.

[5] A.K. Bourke, E.A.F. Ihlen and J.L. Helbostad, Development of a goldstandard method for the identification of sedentary, light and moderate physical activities in older adults: Definitions for video annotation. Journal of Science and Medicine in Sport, vol. 22, iss. 5, 2019, pp. 557561. doi: 10.1016/j.jsams.2018.11.011

[6] R.S. Nagovitsyn, Y.A. Chigovskaya-Nazarova, A.A. Miroshnichenko, and S.Y. Senator, The Realization of the System Programme "Health Saving Education" in the Pedagogical University. European journal of 
[16] Y. Yurchyshyn, Modern information tools: their place in students' extracurriculum and involvement of them in motor activity of health related orientation. Physical Education of Students, vol. 19 (4), 2015, pp. 44-50.

education. Vol. 7, iss. 1, pp. 137-149. doi 10.13187/ejced.2018.1.137

[7] D.H. Yoo, D.S. Ko and I.S. Yeo, Effect of user's trust in usefulness, attitude and intention for mobile sports content services. Journal of Physical Education and Sport, vol. 17(1), 2017, pp. 92-96.

[8] M. Rodrigues, R. Santos, A. Queiros, A.G. Silva, J. Amaral, L.J. Goncalves, A. Pereira, and N.P.D. Rocha, Meet SmartWalk, Smart Cities for Active Seniors. TISHW 2018 - 2nd International Conference on Technology and Innovation in Sports, Health and Wellbeing, Proceedings, 2018, 8559493. doi: 10.1109/TISHW.2018.8559493

[9] S. Torres, P. Simoes, R. Santos, A. Silva and M. Rodrigues, Video content to support physical activity in older adults a strategy for content production centered on user preferences. Iberian Conference on Information Systems and Technologies, CISTI, 2019, 8760724 doi: 10.23919/CISTI.2019.8760724

[10] M.D. Chen, C.C. Kuo, C.A. Pellegrini and M.J. Hsu, Accuracy of wristband activity monitors during ambulation and activities. Med Sci Sports Exerc, vol. 48 (10), 2016, pp. 1942-1949.

[11] A. Henriksen, M.M. Haugen, A.Z. Woldaregay, M. Muzny, G. Hartvigsen, L.A. Hopstock and S. Grimsgaard, Using Fitness Trackers and Smartwatches to Measure Physical Activity in Research: Analysis of Consumer Wrist-Worn Wearables. J Med Internet Res, vol. 20 (3), 2018 e110.

[12] M. Quennerstedt, PE on YouTube - investigating participation in physical education practice. Physical Education and Sport Pedagogy, vol. 18 (1), 2013, pp. 42-59.

[13] J. Escola and J. Jose Applications ICT in Teaching Physical Education. Retos-nuevas tendencias en educacion fisica deporte y recreacion, vol. 34, 2018, pp. 371-376

[14] K.A. Aschbrenner, J.A. Naslund, A.A. Gorin, K.T. Mueser, E.A. Scherer, M. Viron, A. Kinney and S.J. Bartels, Peer support and mobile health technology targeting obesity-related cardiovascular risk in young adults with serious mental illness: Protocol for a randomized controlled trial. Contemporary clinical trials, vol. 74, pp. 97-106, doi: 10.1016/j.cct.2018.10.005

[15] W.E. Kraus, K.F. Janz, K.E. Powell, W.W. Campbell, J.M. Jakicic, R.P. Troiano, K. Sprow, A. Torres and K.L. Piercy, Daily Step Counts for Measuring Physical Activity Exposure and Its Relation to Health Medicine and Science in Sports and Exercise, vol. 51, iss. 6, 2019, pp. 1206-1212.
[17] V. Benavent-Caballer, P. Rosado-Calatayud, E. Segura-Ortí, J.J. AmerCuenca and J.F. Lisón, The effectiveness of a video-supported groupbased Otago exercise programme on physical performance in community-dwelling older adults: a preliminary study. Physiotherapy, vol. 102, iss. 3, 2016, pp. 280-286. doi: 10.1016/j.physio.2015.08.002

[18] V. Kondakov, E. Kopeikina, N. Balysheva, A. Usatov and D. Skrug, Causes of declining interest of students to employment physical education and sports. Physical Education of Students, vol. 19 (1), 2015, pp. 17-21.

[19] S. Alley, S. Schoeppe, D. Guertler, C. Jennings, M.J. Duncan and C. Vandelanotte, Interest and preferences for using advanced physical activity tracking devices: results of a national cross-sectional survey. BMJ Open, 2016, vol. 6 (7), e011243. doi:10.1136/bmjopen-2016011243

[20] O.A. Sinelnikov, Using the iPad in a Sport Education season. Journal of

[21] A. Sushames, A. Edwards, F. Thompson, R. McDermott and K. Gebel, Validity and reliability of Fitbit Flex for step count, moderate to vigorous physical activity and activity energy expenditure. PLoS One, vol. 11 (9), 2016, e0161224. doi: 10.1371/journal.pone.0161224

[22] A.V. Minkin, A.V. Kostin and N.N. Kostina, Mobile devices and software applicable in Russian physical culture and sports GTO complex tests. Teoriya i praktika fizicheskoy kultury, iss. 8, 2017, pp. 34-36

[23] Y. Wahl, P. Düking, A. Droszez, P. Wahl, and J. Mester, CriterionValidity of Commercially Available Physical Activity Tracker to Estimate Step Count, Covered Distance and Energy Expenditure during Sports Conditions. Front Physiol, iss. 8, 2017, 725. doi: 10.3389/fphys.2017.00725

[24] H. Hasan and H. Linger, Enhancing the wellbeing of the elderly: Social use of digital technologies in aged care Educational Gerontology, vol. 42, iss. 11, 2016, pp. 749-757. doi: 10.1080/03601277.2016.1205425

[25] Pauesov S.A. Factors of cadet's physical working capacity dependence in of Physical Education and Sport. 2018, vol. 13(1), pp. 80-87. DOI: 10/14526/01_2018_289 Physical Education, Recreation and Dance, vol. 83 (1), 2012, pp. 39-45. extreme condotions of battle missions realization. The Russian journal 